\title{
Race/ethnicity in systemic AL amyloidosis: perspectives on disease and outcome disparities
}

\author{
Andrew Staron $\mathbb{D}^{1,2}$, Lawreen H. Connors $\mathbb{D}^{1,3}$, Luke Zheng $\mathbb{D}^{4}$, Gheorghe Doros $\mathbb{D}^{1,4}$ and Vaishali Sanchorawala (id)
}

\begin{abstract}
In marked contrast to multiple myeloma, racial/ethnic minorities are underrepresented in publications of systemic light-chain (AL) amyloidosis. The impact of race/ethnicity is therefore lacking in the narrative of this disease. To address this gap, we compared disease characteristics, treatments, and outcomes across racial/ethnic groups in a referred cohort of patients with AL amyloidosis from 1990 to 2020. Among 2416 patients, 14\% were minorities. Non-Hispanic Blacks (NHBs) comprised $8 \%$ and had higher-risk sociodemographic factors. Hispanics comprised $4 \%$ and presented with disproportionately more BU stage IIIb cardiac involvement (27\% vs. 4-17\%). At onset, minority groups were younger in age by 4-6 years. There was indication of more aggressive disease phenotype among NHBs with higher prevalence of difference between involved and uninvolved free light chains $>180 \mathrm{mg} / \mathrm{L}$ (39\% vs. 22-33\%, $P=0.044$ ). Receipt of stem cell transplantation was 30\% lower in Hispanics compared to non-Hispanic White (NHWs) on account of sociodemographic and physiologic factors. Although the age/sex-adjusted hazard for death among NHBs was $24 \%$ higher relative to NHWs $(P=0.020)$, race/ethnicity itself did not impact survival after controlling for disease severity and treatment variables. These findings highlight the complexities of racial/ethnic disparities in AL amyloidosis. Directed efforts by providers and advocacy groups are needed to expand access to testing and effective treatments within underprivileged communities.
\end{abstract}

\section{Introduction}

The incidences of multiple myeloma and its precursor state, monoclonal gammopathy of undetermined significance (MGUS), differ drastically by race/ethnicity. Compared to Whites, rates are over twofold higher in Blacks, slightly higher in Hispanics, and markedly lower in Asians and Pacific Islanders ${ }^{1-5}$. Systemic light-chain (AL) amyloidosis belongs to the spectrum of plasma cell disorders, which include multiple myeloma and MGUS ${ }^{6}$. Accordingly, it would be expected to affect various racial/ ethnic groups in similar disproportions. The largest population-based studies of AL amyloidosis, however, are composed almost entirely of White patients ${ }^{7,8}$. The basis

\footnotetext{
Correspondence: Vaishali Sanchorawala (Vaishali.Sanchorawala@bmc.org) ${ }^{1}$ Amyloidosis Center, Boston University School of Medicine and Boston Medical Center, 1 Boston Medical Center Place, Boston, MA, USA

${ }^{2}$ Section of Hematology and Oncology, Boston University School of Medicine and Boston Medical Center, 1 Boston Medical Center Place, Boston, MA, USA Full list of author information is available at the end of the article
}

for this observation is uncertain. Inequitable access to diagnostic testing and fewer referrals to tertiary centers among disadvantaged groups may be important factorsrather than protective biology.

The Amyloidosis Center at Boston University is situated within a large safety net hospital for the underserved, offering a unique opportunity to evaluate racial/ethnic disparities. In a prior report from our center, emerging recognition of amyloid diseases among underrepresented groups was noted: Blacks doubled in proportion over a three-decade period from 5 to $10 \%$ of patients with systemic AL amyloidosis 9 . To date, little is known about the unique hematologic and organ disease features of this disease in racial/ethnic minorities, and how clinical outcomes may differ. Based on US death certificate data from a national database, mortality due to cardiac amyloid diseases-including both $\mathrm{AL}$ and transthyretin-related (ATTR) amyloidosis-was found to be highest among Black men ${ }^{10}$. In multiple myeloma, racial/ethnic identity 
has an impact on clinical outcomes, largely due to disproportionate use of effective treatments (i.e., stem cell transplantation $)^{11-16}$. With equal access to treatments, survival among Black patients with multiple myeloma is similar or potentially superior compared to Whites ${ }^{2,17-20}$.

Understanding the health obstacles that marginalized patients with hematologic disorders face is necessary to ensure care of equal quality. This article brings new insights on the role of race/ethnicity in systemic AL amyloidosis, including inequities in disease presentation and receipt of aggressive treatment with stem cell transplantation.

\section{Methods}

\section{Data collection and patients}

Data were abstracted from a database of consented patients seen at the Amyloidosis Center at Boston University School of Medicine and Boston Medical Center, which is maintained prospectively under the approval of the Institutional Review Board in accordance with the Declaration of Helsinki and the Health Insurance Portability and Accountability Act Guidelines (ClinicalTrials. gov Identifier: NCT00898235). The diagnosis of AL amyloidosis was predicated on positive staining of tissue with Congo red and evidence of plasma cell clonality in the setting of appropriate systemic syndrome. Multiple myeloma-associated AL amyloidosis signified cases that also fulfilled the International Myeloma Working Group criteria for multiple myeloma, including both myelomadefining end-organ features and clonal bone marrow plasma cell requirements, which changed over the course of the study period. In view of the high prevalence of hereditary ATTR amyloidosis among individuals of African ancestry ${ }^{21,22}$, self-reported Black patients underwent screening for the transthyretin V122I genetic variant using isoelectric focusing ${ }^{23}$ and, when appropriate, mutation confirmation by direct DNA-sequencing ${ }^{21}$. Mass spectrometric analysis of tissue confirmed the presence of AL rather than ATTR amyloidosis in cases where the V122I allele was detected.

Sociodemographic (educational level, marital status) and lifestyle (alcohol consumption, smoking status, body mass index (BMI)) factors, along with baseline hematologic and organ characteristics, were stratified according to self-identified race/ethnicity. Groups were mutually exclusive and included non-Hispanic White (NHW, i.e., White alone), non-Hispanic Black (NHB, i.e., African American, Afro-Caribbean, or Black alone), Hispanic (i.e., Hispanic or Latinx, any race), and non-Hispanic other (NHO, i.e., Asian, Pacific Islander, American Indian, Alaskan Native, more than one race, or unidentified).

\section{Staging and response assessment}

Organ involvement was defined according to the consensus criteria of the International Society of
Amyloidosis $^{24}$. Cardiac staging was established on brain natriuretic peptide (BNP, threshold $>81 \mathrm{pg} / \mathrm{mL}$ ) and troponin-I (threshold $>0.1 \mathrm{ng} / \mathrm{mL}$ ) with stages I, II, and III defined according to none, one or both markers above threshold ${ }^{25}$. Stage IIIb was classified if BNP was also $>700 \mathrm{pg} / \mathrm{mL}$. Similarly, renal staging was based on estimated glomerular filtration rate by Chronic Kidney Disease Epidemiology Collaboration (CKD-EPI) creatinine equation (threshold $<50 \mathrm{~mL} / \mathrm{min}$ per $1.73 \mathrm{~m}^{2}$ ) and $24-\mathrm{h}$ proteinuria (threshold $>5 \mathrm{~g} / 24 \mathrm{~h})^{26}$. For patients who returned to our center for follow-up evaluation, interim treatments received were recorded, and hematologic response defined according to a validated criterion using immunofixation electrophoresis and serum free lightchain ratio ${ }^{27}$.

\section{Statistical analysis}

Where present, incomplete data were reviewed and determined missing at random (i.e., due to variable entry not yet built into the database, unavailability of laboratory test, or data point not ascertained during history taking from patient) and a complete-case approach was taken. The number of complete cases $(N)$ for those variables with missing data was indicated in the tables. For baseline descriptors, categorical variables were presented as proportions and differences assessed by the $\chi^{2}$ test of independence. Continuous data were presented as medians with interquartile ranges (IQRs) and comparisons made by the one-way analysis of variance test. Multivariable logistic regression was used to evaluate the confounding effect of sociodemographic and physiologic variables on stem cell transplantation utilization. Overall survival was defined as time from tissue diagnosis to date of death from any cause (event) or last follow-up (censored). Unadjusted survival curves were plotted by Kaplan-Meier method. Hazard ratios (HRs) for death and 95\% confidence intervals (CIs) were estimated using multivariate Cox proportional hazards regression models in order to determine the effect of race/ethnicity on mortality while adjusting for relevant confounders. Initially, only demographic variables were included (model 1). Sociodemographic and lifestyle factors were then added (model 2), followed by physiologic factors (model 3). Lastly, stem cell transplantation treatment was included (model 4). $P$ values were two-sided with $<0.05$ considered statistically significant. Computations were performed with the SAS version 9.4 software.

\section{Results}

A total of 2416 patients with systemic AL amyloidosis were evaluated between January 1, 1990 and January 1, 2020, including 2078 (86\%) NHW, 192 (8\%) NHB, 87 (4\%) Hispanic, and 59 (2\%) NHO individuals (Fig. 1a). Underrepresented minorities (NHB, Hispanic, and NHO) 


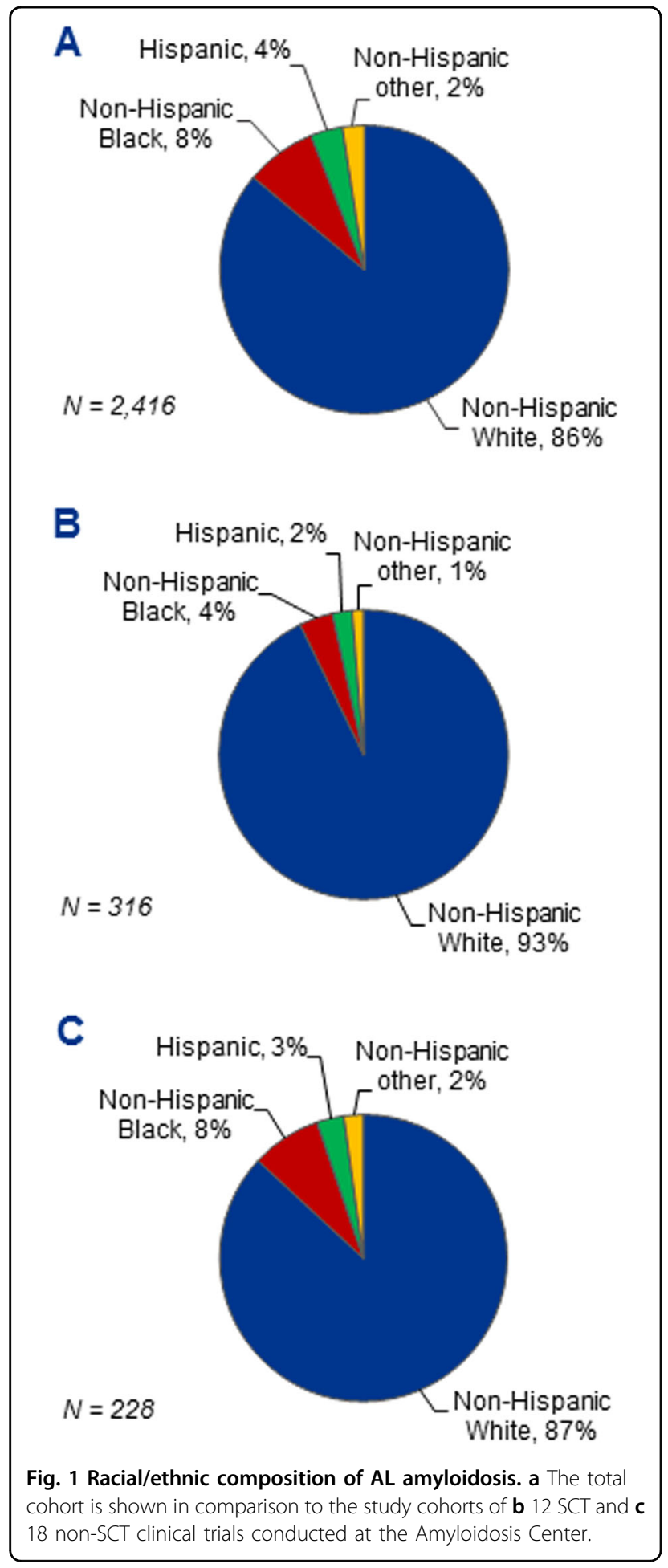

comprised an increasing proportion of the population over time: $8 \%$ in $1990-1999 ; 13 \%$ in $2000-2009$; and $19 \%$ in 2010-2020. NHB and Hispanic patients were more likely to be local referrals (i.e., from the state of Massachusetts, Table 1). Median age was significantly different between groups $(P<0.001)$ with $\mathrm{NHOs}$ diagnosed the youngest at 56 years and NHWs the oldest at 62 years. Sex distribution was similar across groups with males comprising $54-59 \%$ of the cohort.

\section{Sociodemographic and lifestyle factors}

Table 1 compares sociodemographic and lifestyle factors. Among Hispanics and NHBs, educational attainment was significantly lower with high school level or less in $45 \%$ and $39 \%$ of patients, respectively, compared to $25 \%$ among NHWs $(P<0.001)$. Proportionally, NHWs and NHOs had the most married patients, whereas NHBs had the most single patients $(P<0.001)$. Alcohol consumption was highest among NHWs ( $58 \%$ vs. $32-45 \%$ of patients, $P<0.001)$. Differences in cigarette smoking status and intensity were non-significant. Regarding BMI, overweight BMI (25-29.9) was most prevalent among Hispanics and obese BMI $(\geq 30)$ among NHBs, whereas normal BMI (18.5-24.9) was most prevalent among NHOs $(P<0.001)$.

\section{Hematologic parameters}

When stratified by race/ethnicity, no significant differences were found in the distribution of $\mathrm{AL}$ amyloidosis types (i.e., $\mathrm{AL}$ alone vs. multiple myeloma-associated $\mathrm{AL}$ ) or the involved light-chain category (i.e., $\lambda$ vs. $\kappa$ ) as shown in Table 2. Notably, all cases of Waldenström macroglobulinemia-associated AL amyloidosis occurred in the NHW group, with the exception of one NHB patient. While the median difference between involved (amyloidogenic) and uninvolved free light chains (dFLC) at baseline did not differ significantly by race/ethnicity, NHBs had a considerably higher proportion of patients with $\mathrm{dFLC}>180 \mathrm{mg} / \mathrm{L}$ (39\% vs. $22-33 \%, P=0.044)$.

\section{Organ disease characteristics}

Cardiac involvement was most prevalent among NHBs (69\% of patients) and renal involvement among NHWs (78\% of patients), although differences in pattern of organ involvement across racial/ethnic groups did not meet statistical thresholds (Table 3). Most striking, Hispanics were found to have a significantly higher median BNP level at baseline compared to the other groups (1041 vs. $221-480 \mathrm{pg} / \mathrm{mL}, P=0.001$ ) with the majority having a BNP level $>700 \mathrm{pg} / \mathrm{mL}$. This corresponded with a higher proportion of Hispanics having BU cardiac stage IIIb disease compared to NHWs ( $27 \%$ vs. $15 \%$ of patients, $P=$ 0.027 by Fisher's exact test), although the distribution of all cardiac stages did not differ between these two groups $(P=0.080)$. Meanwhile, NHOs had lower-stage cardiac involvement. The distribution of renal stages was similar between groups $(P=0.113)$. In this population, time intervals from first patient-reported sign or symptom (related to any involved organ) to diagnosis did not differ 
Table 1 Sociodemographic and lifestyle factors by race/ethnicity.

\begin{tabular}{|c|c|c|c|c|c|}
\hline & NHW & NHB & Hispanic & $\mathrm{NHO}$ & $P$ value \\
\hline$N($ total $=2416)$ & 2078 & 192 & 87 & 59 & \\
\hline Median age, years (IQR) & $62(54-69)$ & $58(50-66)$ & $58(52-67)$ & $56(45-65)$ & $<0.001$ \\
\hline \multicolumn{6}{|l|}{ Sex, $n(\%)$} \\
\hline Male & $1229(59 \%)$ & $114(59 \%)$ & $48(55 \%)$ & $32(54 \%)$ & \\
\hline Female & $849(41 \%)$ & $78(41 \%)$ & $39(45 \%)$ & $27(46 \%)$ & 0.778 \\
\hline \multicolumn{6}{|l|}{ Geographic origin, $n$ (\%) } \\
\hline Massachusetts (local) & $371(18 \%)$ & $47(25 \%)$ & $28(32 \%)$ & $8(14 \%)$ & $<0.001$ \\
\hline Outside of New England & $1358(65 \%)$ & $131(68 \%)$ & $52(60 \%)$ & 47 (80\%) & 0.068 \\
\hline \multicolumn{6}{|c|}{ Educational level $(N=1955), n(\%)$} \\
\hline Postsecondary & $1261(75 \%)$ & $92(61 \%)$ & $36(56 \%)$ & $41(76 \%)$ & \\
\hline High school or less & $425(25 \%)$ & $58(39 \%)$ & $29(45 \%)$ & $13(24 \%)$ & $<0.001$ \\
\hline \multicolumn{6}{|c|}{ Marital status $(N=2249), n(\%)$} \\
\hline Married & $1526(80 \%)$ & $128(67 \%)$ & $65(75 \%)$ & $46(81 \%)$ & \\
\hline Single & $124(6 \%)$ & $35(18 \%)$ & $9(10 \%)$ & $3(5 \%)$ & \\
\hline Separated or widowed & $265(14 \%)$ & $27(14 \%)$ & $13(15 \%)$ & $8(14 \%)$ & $<0.001$ \\
\hline \multicolumn{6}{|c|}{ Alcohol consumption $(N=2346), n(\%)$} \\
\hline No use & $855(42 \%)$ & $123(67 \%)$ & $48(55 \%)$ & $40(68 \%)$ & \\
\hline Occasional use & $927(46 \%)$ & $52(28 \%)$ & $34(39 \%)$ & 17 (29\%) & \\
\hline Moderate-to-heavy use & $234(12 \%)$ & $9(5 \%)$ & $5(6 \%)$ & $2(3 \%)$ & $<0.001$ \\
\hline \multicolumn{6}{|c|}{ Smoking status $(N=2267), n(\%)$} \\
\hline Never smoker & 1097 (57\%) & $113(61 \%)$ & $50(60 \%)$ & $43(73 \%)$ & \\
\hline Current or former smoker & $843(43 \%)$ & $72(39 \%)$ & $33(40 \%)$ & $16(27 \%)$ & 0.054 \\
\hline Median pack years (IQR) & $15(4-35)$ & $10(2-30)$ & $20(4-30)$ & $8(1-30)$ & 0.117 \\
\hline \multicolumn{6}{|l|}{ BMI class $(N=2044), n(\%)$} \\
\hline Normal (18.5-24.9) & $656(38 \%)$ & $68(39 \%)$ & $28(37 \%)$ & $34(64 \%)$ & \\
\hline Overweight (25-29.9) & $693(40 \%)$ & $50(29 \%)$ & $35(46 \%)$ & 17 (32\%) & \\
\hline Obese $(\geq 30)$ & $391(22 \%)$ & $57(33 \%)$ & $13(17 \%)$ & $2(4 \%)$ & $<0.001$ \\
\hline Median BMI, kg/m² (IQR) & $26(23-30)$ & $27(24-31)$ & $27(24-28)$ & $24(22-27)$ & $<0.001$ \\
\hline
\end{tabular}

NWH non-Hispanic White, NHB non-Hispanic Black, NHO non-Hispanic other, IQR interquartile range, $B M I$ body mass index.

among groups, nor did time intervals from diagnosis to initial visit at the Amyloidosis Center (Table 3).

\section{Receipt of stem cell transplantation}

The use of high-dose melphalan and autologous stem cell transplantation (HDM/SCT) was evaluated in a subcohort of 1668 patients for whom a plasma cell-directed treatment was documented. Differences were notable, although non-significant $(P=0.071)$, between racial/ethnic groups (Fig. 2). Receipt of HDM/SCT was lowest in the Hispanic group with 19 (33\%) patients transplanted, compared to $680(47 \%)$ in the NHW, 48 (39\%) in the
$\mathrm{NHB}$, and 18 (42\%) in the NHO groups. A multivariable logistic regression, which adjusted for the effect of confounding risk factors on HDM/SCT utilization, revealed that lower educational attainment (i.e., high school level or less) and later-stage cardiac involvement (i.e., BU cardiac stage III/IIIb) significantly reduced the odds of receiving $\mathrm{HDM} / \mathrm{SCT}$, but not race/ethnicity itself. In terms of post-transplantation outcomes, rates of hematologic complete response (hemCR, assessed at 6-12 months after HDM/SCT) were non-significantly different for minority groups compared to NHWs (44-58\% vs. $37 \%, P=0.562$ ). 
Table 2 Comparison of hematologic parameters by race/ethnicity.

\begin{tabular}{|c|c|c|c|c|c|}
\hline & NHW & NHB & Hispanic & $\mathrm{NHO}$ & $P$ value \\
\hline \multicolumn{6}{|c|}{ AL amyloidosis type, $n(\%)$} \\
\hline AL alone & $1862(90 \%)$ & $171(89 \%)$ & $76(87 \%)$ & $55(93 \%)$ & \\
\hline MM-associated AL & $154(7 \%)$ & $20(10 \%)$ & $10(12 \%)$ & $3(5 \%)$ & \\
\hline Other $^{a}$ & $62(3.0 \%)$ & $1(<1 \%)$ & $1(1 \%)$ & $1(2 \%)$ & 0.155 \\
\hline \multicolumn{6}{|c|}{ Involved light chain $(N=2280), n(\%)$} \\
\hline k & $456(23 \%)$ & $39(21 \%)$ & $20(24 \%)$ & $13(22 \%)$ & \\
\hline$\lambda$ & $1492(77 \%)$ & $150(79 \%)$ & $65(76 \%)$ & $45(78 \%)$ & 0.857 \\
\hline \multicolumn{6}{|l|}{$\mathrm{dFLC}^{\mathrm{b}}(N=1640)$} \\
\hline Median, mg/L (IQR) & $89(27-269)$ & $116(23-310)$ & $76(24-168)$ & $90(29-177)$ & 0.416 \\
\hline$\geq 180 \mathrm{mg} / \mathrm{L}, n(\%)$ & $455(33 \%)$ & $54(39 \%)$ & $16(24 \%)$ & $11(22 \%)$ & 0.044 \\
\hline
\end{tabular}

$M M$ multiple myeloma, $d F L C$ difference between involved and uninvolved free light chains, IQR interquartile range.

a Other types included Waldenström macroglobulinemia-associated or lymphoplasmacytic lymphoma-associated AL amyloidosis.

${ }^{\mathrm{b}}$ The serum free light-chain assay was unavailable before 2003.

\section{Participation in clinical trials}

Between 1990 and 2020, there were 12 clinical trials of SCT and 18 of non-SCT therapies for patients with AL amyloidosis conducted at the center. Of 316 patients enrolled on SCT clinical trials (Fig. 1b), 295 (93\%) were NHW, 12 (4\%) were NHB, 7 (2\%) were Hispanic, and 4 (1\%) were NHO. Similarly, of 228 patients enrolled on non-SCT clinical trials (Fig. 1c), 199 (87\%) were NHW, 18 (8\%) were NHB, 7 (3\%) were Hispanic, and 5 (2\%) were NHO. Some patients participated in multiple trials. Collectively, representation of racial/ethnic minorities in all trials was $10 \%$. This improved more recently, as demonstrated by the 13 trials introduced in the latest decade (2010-2020), in which 25 (22\%) of 116 patients were minorities.

\section{Survival}

At the time of data cutoff (March 2020), 1622 (67\%) patients were deceased. Kaplan-Meier survival curves according to race/ethnicity are illustrated in Fig. 3. Among the groups, NHBs had the shortest median overall survival (2.5 vs. 3.4-7.9 years). Early mortality was disproportionately higher among NHBs with 23\% (95\% CI, 18-30\%) of patients deceased within 6 months of diagnosis, compared to $15 \%$ (95\% CI, 13-17\%) among NHWs. Multivariate Cox proportional analysis demonstrated no significance in the overall effect of race/ethnicity on mortality; however, slight differences among specific groups were observed. The influence of various patient characteristics was accounted for in stepwise fashion (Table 4). Unadjusted, NHBs had a 15\% higher HR for death than NHWs $(P=0.145)$. After adjusting for age and sex, this disparity intensified to $24 \%$ and became significant $(P=0.020)$. Adding sociodemographic and lifestyle variables to the model reduced the disparity slightly, whereas adding physiologic variables and HDM/ SCT treatment diminished it entirely. The variables with the strongest effect size on mortality risk in our final analysis (model 4) were HDM/SCT receipt (HR, 0.45; 95\% CI, 0.33-0.60; $P<0.001)$ and cardiac stage $\geq$ III (HR, 1.94; 95\% CI 1.48-2.54; $P<0.001)$, followed by dFLC $>180 \mathrm{mg} /$ mL (HR, 1.39; 95\% CI, 1.06-1.82; $P=0.015$ ).

\section{Discussion}

While efforts have been made to understand racial/ ethnic disparities in multiple myeloma and MGUS, the first steps need yet to be taken for systemic AL amyloidosis. In our analysis of 2416 patients, 334 (14\%) were self-identified minorities. This percentage is considerably lower than the $>36 \%$ representation of $\mathrm{racial} / \mathrm{ethnic}$ minorities in the general population (based on 2010 US census data $)^{28}$. The discrepancy was found to be most profound for Hispanics, who constitute $>16 \%$ of the general population, but only represented $4 \%$ of the AL amyloidosis cohort. Although these data depict the experience of a single-referral center, the findings are compelling. Systematic underdetection may be at the root of the disparity, perhaps due in part to the misattribution of AL indicators (e.g., structural heart changes, nephrosis, neuropathy) to hypertensive disease or diabetes mellitusboth of which are more prevalent in racial/ethnic minorities.

Disparities in access to diagnostic testing and specialized treatment centers often reflect socioeconomic obstacles, which vary by race/ethnicity ${ }^{29}$. It was therefore important to contextualize the social determinants of health in our cohort. More adverse risk factors were observed among underrepresented minorities, including 
Table 3 Organ disease characteristics by race/ethnicity.

\begin{tabular}{|c|c|c|c|c|c|}
\hline & NHW & NHB & Hispanic & NHO & $P$ value \\
\hline \multicolumn{6}{|l|}{ Organ involvement, $n$ (\%) } \\
\hline Heart $(N=1963)$ & $1010(62 \%)$ & $124(69 \%)$ & $56(67 \%)$ & $29(54 \%)$ & 0.122 \\
\hline Kidney $(N=2121)$ & 1397 (78\%) & $134(72 \%)$ & $61(72 \%)$ & $37(69 \%)$ & 0.068 \\
\hline Liver $(N=1846)$ & $422(27 \%)$ & $57(32 \%)$ & $21(27 \%)$ & $13(25 \%)$ & 0.508 \\
\hline Nervous system ${ }^{\mathrm{a}}(N=1806)$ & $566(38 \%)$ & $51(30 \%)$ & $28(35 \%)$ & $13(27 \%)$ & 0.097 \\
\hline \multicolumn{6}{|l|}{ Organ biomarkers } \\
\hline $\mathrm{BNP}(\mathrm{N}=1338), \mathrm{pg} / \mathrm{mL}(\mathrm{IQR})$ & $424(206-881)$ & $480(207-863)$ & $1041(429-1758)$ & $221(107-510)$ & 0.001 \\
\hline $\mathrm{BNP}>700 \mathrm{pg} / \mathrm{mL}, n(\%)$ & $212(33 \%)$ & $27(33 \%)$ & $22(61 \%)$ & $4(17 \%)$ & 0.002 \\
\hline \multicolumn{6}{|l|}{ BU cardiac stage ${ }^{\mathrm{b}}(N=1158), n(\%)$} \\
\hline Stage I & $267(29 \%)$ & $30(26 \%)$ & $18(30 \%)$ & $21(45 \%)$ & \\
\hline Stage II & $400(43 \%)$ & $48(41 \%)$ & $18(30 \%)$ & $21(45 \%)$ & \\
\hline Stage III & $126(14 \%)$ & $19(16 \%)$ & $8(13 \%)$ & $3(6 \%)$ & \\
\hline Stage IIIb & $141(15 \%)$ & $20(17 \%)$ & $16(27 \%)$ & $2(4 \%)$ & 0.033 \\
\hline Pairwise comparison, $P$ & Ref. & 0.718 & 0.080 & 0.024 & \\
\hline Proteinuria $(N=2221), g / 24 \mathrm{~h}(\mathrm{IQR})$ & $1195(105-6027)$ & $1164(183-4447)$ & $486(93-4592)$ & $781(103-5628)$ & 0.059 \\
\hline eGFR $(N=2088), \mathrm{mL} / \mathrm{min}$ per $1.73 \mathrm{~m}^{2}(\mathrm{IQR})$ & $68(39-91)$ & $73(35-106)$ & $64(21-89)$ & $90(59-102)$ & $<0.001$ \\
\hline \multicolumn{6}{|l|}{ Renal stage $(N=1932), n(\%)$} \\
\hline Stage I & $787(48 \%)$ & 77 (52\%) & $33(45 \%)$ & $31(57 \%)$ & \\
\hline Stage ॥ & $653(39 \%)$ & $61(42 \%)$ & $34(47 \%)$ & $18(33 \%)$ & \\
\hline Stage III & $218(13 \%)$ & $9(6 \%)$ & $6(8 \%)$ & $5(9 \%)$ & 0.113 \\
\hline Alkaline phosphatase $(N=2077)$, IU/L (IQR) & $92(71-135)$ & $103(71-152)$ & $98(76-180)$ & $100(74-149)$ & 0.841 \\
\hline \multicolumn{6}{|l|}{ Diagnostic and referral intervals } \\
\hline $\begin{array}{l}\text { Time from first reported sign/symptom to diagnosis in months } \\
(N=1933) \text {, median (IQR) }\end{array}$ & $6.8(2.3-13.5)$ & $6.2(1.7-12.7)$ & $4.1(1.5-9.9)$ & $5.2(2.0-12.8)$ & 0.333 \\
\hline $\begin{array}{l}\text { Time from diagnosis to initial visit at the referral center in months, } \\
\text { median (IQR) }\end{array}$ & $2.5(1.4-6.0)$ & $2.9(1.4-6.4)$ & $2.0(1.1-4.1)$ & $3.6(1.6-9.7)$ & 0.603 \\
\hline
\end{tabular}

Laboratory measurements during initial evaluation is presented here as medians with IQRs.

$B N P$ brain natriuretic peptide, BU Boston University, Ref. reference group, eGFR estimated glomerular filtration rate by CKD-EPI equation, IQR interquartile range. ${ }^{a}$ Denotes autonomic and/or peripheral nervous system.

${ }^{\mathrm{b}}$ BNP and troponin-I were unavailable before the mid-2000s.

lower educational attainment and a greater proportion of unmarried individuals. Independently, these factors have important implications for health and survival. Significant differences were also noted in BMI classification-a potentially modifiable risk factor-with the Hispanic and NHB groups having disproportionately more overweight/ obese individuals. Obesity and its associated low-grade inflammatory state are thought to alter plasma cell development, leading to a nearly twofold higher risk for developing MGUS and progressing to overt multiple myeloma $^{30-33}$. Particularly among NHBs, higher body weight is associated with an excess risk of multiple myeloma-related mortality ${ }^{34}$. In $\mathrm{AL}$ amyloidosis, the role of obesity on pathogenesis of the underlying plasma cell neoplasm and/or downstream organ disease severitywhich may especially impact minorities-is not yet defined.

Importantly, a number of differences in disease features were uncovered in our study. Racial/ethnic minorities were diagnosed at younger ages compared to NHWs (e.g., 4 years younger for NHBs), an observation that is paralleled in other plasma cell disorders ${ }^{2,19}$. This age difference was not explained by lifestyle risk exposures (i.e., alcohol use and cigarette smoking were lower or similar among minorities). Furthermore, the NHB group had disproportionately more patients with a baseline dFLC $>180 \mathrm{mg} / \mathrm{L}$, which is thought to reflect a more aggressive disease phenotype ${ }^{6}$. At face value, these findings may 
- HDM/SCT utilized ${ }^{*}$ - HemCR rate among HDM/SCT recipient ${ }^{\star}$

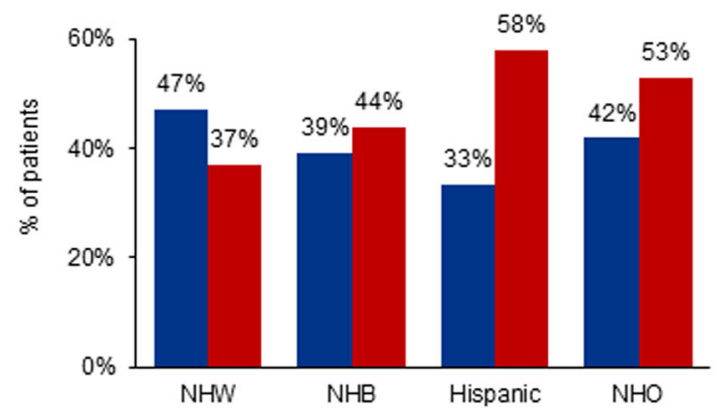

Factors predicting HDM/SCT utilization

\begin{tabular}{lcc}
\multicolumn{3}{l}{ Factors predicting HDM/SCT utilization } \\
\hline Variable & OR $(95 \% \mathrm{Cl})$ & $\mathrm{P}$ \\
\hline NHW & ref & \\
NHB & $0.81(0.43-1.50)$ & .498 \\
Hispanic & $1.08(0.42-2.77)$ & .871 \\
NHO & $0.61(0.27-1.40)$ & .243 \\
Older age at diagnosis & $0.93(0.91-0.95)$ & $<.001$ \\
Sex (female vs. male) & $0.86(0.59-1.26)$ & .442 \\
Education (high school or less vs. & $0.49(0.31-0.76)$ & .002 \\
postsecondary) & & \\
Marital status (single vs. married) & $1.07(0.54-2.12)$ & .857 \\
BU cardiac stage (III/IIb vs. I/II) & $0.33(0.21-0.52)$ & $<.001$ \\
Renal stage (III vs. I/II) & $1.06(0.61-1.84)$ & .847 \\
dFLC > 180 mg/L & $0.87(0.57-1.33)$ & .519
\end{tabular}

Fig. 2 Use of HDM/SCT and post-transplantation outcomes.

*Based on a subcohort of 1668 patients with available treatment data, of whom 765 received HDM/SCT. Differences in utilization were nonsignificant $(P=0.071)$. ${ }^{\dagger}$ Differences in hematologic complete response (hemCR) rates were also non-significant $(P=0.562)$. A multivariable logistic regression analysis of HDM/SCT utilization is provided. HDM/ SCT high-dose melphalan and autologous stem cell transplantation, NHW non-Hispanic White, ref reference group, NHB non-Hispanic Black, NHO non-Hispanic other, OR odds ratio, BU Boston University, dFLC difference between involved and uninvolved free light chains.



Fig. 3 Kaplan-Meier survival curves stratified by race/ethnicity. NHW non-Hispanic White, NHB non-Hispanic Black, NHO nonHispanic other.

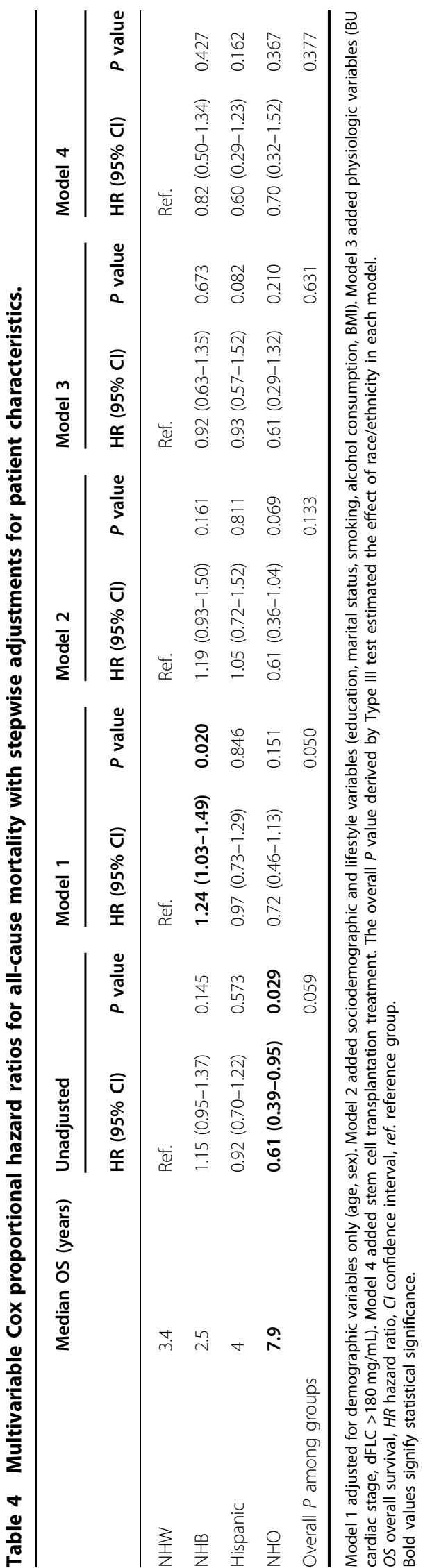


imply unique disease physiology, and future investigations are needed to unravel the potential biologic and/or genetic underpinnings of ancestry-related disparities in $\mathrm{AL}$ amyloidosis. Alternatively, these findings may be explained by underdetection of NHB individuals with more indolent disease-instead necessitating heightened awareness.

Organ distribution was similar across racial/ethnic groups, yet differences in organ severity were noted. The most advanced cardiac stage (i.e., BU stage IIIb) was 1.8fold higher among Hispanics compared to NHWs-likely a reflection of delayed disease recognition. Although the time interval from first patient-reported symptom to diagnosis was estimated not to be longer among Hispanics, it may be that some minorities underreport symptoms-due in part to language barriers-or seek medical attention later. This disparity in organ manifestation may also signal differences in the amyloidogenic light chain. While little is known about the mechanisms of organ tropism and proteotoxicity in AL amyloidosis, structural and genetically-driven features of the immunoglobulin light-chain variable region (IGLV) have been implicated. For example, germline expression of the IGLV1-44 gene has been associated with cardiac tropism, whereas the IGLV6-57 gene with renal tropism ${ }^{35-37}$. Variances in this gene have not before been compared across racial/ethnic populations.

When evaluating cardiac amyloidosis in persons of African ancestry, hereditary ATTR amyloidosis needs to be excluded before a diagnosis of AL amyloidosis can be established. The G>A nucleotide transition at the first base position in codon 122 of the transthyretin gene (encoding for TTR V122I) has a frequency of 3.4\% among African Americans, and is a major contributor of ATTR cardiomyopathy $^{22}$. Notably, ATTR V122I and AL amyloidosis were previously found to be equally prevalent as causes of amyloid cardiomyopathy in African Americans evaluated at our center ${ }^{21}$. One further challenge in establishing diagnosis is the coexistence of MGUS in nearly $50 \%$ of African Americans with ATTR V122I amyloidosis $^{38}$. In our study, 4 (2\%) of 179 patients with $\mathrm{AL}$ amyloidosis in the NHB group tested were found to be carriers of the V122I variant allele. Penetrance is $<100 \%$ in hereditary ATTR amyloidosis; thus, not every patient who tests positive for the V122I variant allele has or will develop ATTR V122I amyloidosis. In this situation, typing of amyloid protein is essential prior to treatment. Moreover, an appropriate systemic syndrome is a requisite for AL amyloidosis, as cases of cardiac amyloid fibrils containing both light-chain and TTR proteins have recently been described ${ }^{39}$.

Despite an evolving therapeutic landscape for $\mathrm{AL}$ amyloidosis, HDM/SCT remains one of the most effective treatments available ${ }^{40,41}$. In our study, racial/ethnic minorities were less likely to receive treatment with $\mathrm{HDM} / \mathrm{SCT}$, with Hispanics in particular having a 30\% lower likelihood compared to NHWs. Similar patterns have been reported in multiple myeloma, contributing to higher mortality risk ${ }^{11-13,42}$. Identifying and mitigating treatment barriers is therefore important. We found that sociodemographic (e.g., lower educational attainment) and physiologic (e.g., cardiac stage) factors were the key drivers of HDM/SCT underutilization, rather than race/ ethnicity itself. This finding underscores the need for earlier disease detection in underrepresented communities-before cardiac disease becomes too advanced to qualify for HDM/SCT treatment. Moreover, tackling upstream economic barriers, expanding health literate resources, and funding care coordination programs may help bridge access to treatment with HDM/SCT. Race/ ethnicity did not impact post-transplantation outcomes. A larger sample size would be needed to determine whether the $6-21 \%$ higher rates of hemCR among minorities observed in this study are significant.

Enrollment in therapeutic clinical trials can provide access to advanced treatments. Most published trials of AL amyloidosis do not report the racial/ethnic construct of the study cohort. We retrospectively evaluated the accrual of racial/ethnic minorities in 30 clinical trials conducted at our center and observed that minorities comprised only $10 \%$ of study participants. Underrepresentation of minorities in clinical trials limits understanding about the safety and efficacy of promising treatments across populations, and thus has the potential to contribute to racial/ethnic disparities ${ }^{43}$. Multilevel interventions are needed, including attentiveness by investigators to recruit minorities and engagement of institutions serving minority communities in trials of $\mathrm{AL}$ amyloidosis.

Timely diagnosis and treatment are important in order to reduce early mortality and halt organ progression in $\mathrm{AL}$ amyloidosis $^{6}$. Within this referred cohort, NHBs had the highest rate of early mortality and shortest overall survival. In view of the age difference at diagnosis, mortality risk was adjusted for demographic variables and found to be significantly greater among NHBs compared to NHWs. This survival disparity was eliminated when markers of disease severity (i.e., cardiac stage and dFLC) were taken into account, indicating that race itself did not impact survival. Interestingly, Hispanics were not found to have inferior survival despite more patients having stage IIIb cardiac involvement-perhaps due to insufficient sample size to detect a difference or more indolent disease phenotype in this population.

There are several limitations of our study. Race and ethnicity are sociocultural-rather than biological-constructs. Self-reported racial/ethnic category can therefore be a biased measure when evaluating differences in 
disease characteristics. Another inherent limitation is referral bias. Underprivileged individuals face greater difficulties in accessing tertiary centers, and thus may remain uncaptured in our study. Large population databases of AL amyloidosis apart from referral centers, however, are nonexistent. Furthermore, the unavailability of a direct measure of socioeconomic status in our database-which is an important influence in studies of race/ ethnicity-required us to depend on surrogates, such as educational level. Despite these limitations, this study sheds light on the clinical presentation and outcomes of minority patients with AL amyloidosis who are underrepresented in the medical literature to date.

\section{Conclusions}

Racial/ethnic minorities comprised a small portion of patients with systemic AL amyloidosis seen at a referral center, drawing attention to systematic barriers in health care access. The minorities captured in this study already overcame many of the obstacles that underlie disparities. Nonetheless, earlier and more severe diagnosis was detected among NHBs and Hispanics. Cardiac staging and educational level accounted for underutilization of HDM/ $\mathrm{SCT}$, rather than race/ethnicity itself. After controlling for disease severity and HDM/SCT treatment, survival did not differ by race/ethnicity. Greater awareness is needed, along with innovative approaches to make services and technologies for earlier identification of AL amyloidosis available in underrepresented communities.

\section{Acknowledgements}

The Amyloidosis Center database and repository are supported by the Amyloid Research Fund of Boston University School of Medicine. We acknowledge the vision of the founding director, Dr. Martha Skinner, in maintaining the database and thank members of the multidisciplinary team for the care of patients.

\section{Author details \\ ${ }^{1}$ Amyloidosis Center, Boston University School of Medicine and Boston Medical Center, 1 Boston Medical Center Place, Boston, MA, USA. ${ }^{2}$ Section of Hematology and Oncology, Boston University School of Medicine and Boston Medical Center, 1 Boston Medical Center Place, Boston, MA, USA. ${ }^{3}$ Department of Pathology and Laboratory Medicine, Boston University School of Medicine and Boston Medical Center, 1 Boston Medical Center Place, Boston, MA, USA. ${ }^{4}$ Department of Biostatistics, Boston University School of Public Health, 715 Albany Street, Boston, MA, USA}

\section{Author contributions}

A.S.: Conceptualization and design, collection and assembly of data, data analysis and interpretation, writing of manuscript. L.H.C.: Curation of data from database. L.Z. and G.D.: Statistical analysis and interpretation. V.S.: Conceptualization, supervision and approval of final draft. All authors provided critical revisions to the manuscript.

\section{Conflict of interest}

The authors declare that they have no conflict of interest.

\section{Publisher's note}

Springer Nature remains neutral with regard to jurisdictional claims in published maps and institutional affiliations.
Supplementary Information accompanies this paper at (https://doi.org/ 10.1038/s41408-020-00385-0)

Received: 7 July 2020 Revised: 1 October 2020 Accepted: 23 October 2020 Published online: 10 November 2020

\section{References}

1. Landgren, $\mathrm{O}$. et al. Risk of monoclonal gammopathy of undetermined significance (MGUS) and subsequent multiple myeloma among African American and White veterans in the United States. Blood 107, 904-906 (2006).

2. Waxman, A. J. et al. Racial disparities in incidence and outcome in multiple myeloma: a population-based study. Blood 116, 5501-5506 (2010).

3. Landgren, O. et al. Racial disparities in the prevalence of monoclonal gammopathies: a population-based study of 12,482 persons from the National Health and Nutritional Examination Survey. Leukemia 28, 1537-1542 (2014).

4. Landgren, $\mathrm{O}$. et al. Prevalence of myeloma precursor state monoclonal gammopathy of undetermined significance in 12372 individuals 10-49 years old: a population-based study from the National Health and Nutrition Examination Survey. Blood Cancer J. 7, e618 (2017).

5. Howlader, N. et al. SEER Cancer Statistics Review, 1975-2017 (National Cancer Institute, Bethesda, 2019).

6. Merlini, G. et al. Systemic immunoglobulin light chain amyloidosis. Nat. Rev. Dis. Primers 4, 38 (2018).

7. Kyle, R. A. et al. Incidence of AL amyloidosis in Olmsted County, Minnesota, 1990 through 2015. Mayo Clin. Proc. 94, 465-471 (2019).

8. Pinney, J. H. et al. Systemic amyloidosis in England: an epidemiological study. Br. J. Haematol. 161, 525-532 (2013).

9. Staron, A., Connors, L. H., Ruberg, F. L., Mendelson, L. M. \& Sanchorawala, V. A new era of amyloidosis: the trends at a major US referral centre. Amyloid 26, 192-196 (2019).

10. Alexander, K. M. et al. Geographic disparities in teported US amyloidosis mortality from 1979 to 2015: potential underdetection of cardiac amyloidosis. JAMA Cardiol. 3, 865-870 (2018).

11. Fiala, M. A. \& Wildes, T. M. Racial disparities in treatment use for multiple myeloma. Cancer 123, 1590-1596 (2017).

12. Schriber, J. R. et al. Hispanics have the lowest stem cell transplant utilization rate for autologous hematopoietic cell transplantation for multiple myeloma in the united states: a CIBMTR report. Cancer 123, 3141-3149 (2017).

13. Ailawadhi, S. et al. Racial disparities in treatment patterns and outcomes among patients with multiple myeloma: a SEER-Medicare analysis. Blood Adv 3, 2986-2994 (2019).

14. Marinac, C. R., Ghobrial, I. M., Birmann, B. M., Soiffer, J. \& Rebbeck, T. R. Dissecting racial disparities in multiple myeloma. Blood Cancer J. 10, 19 (2020).

15. Dennis, M. et al. Treatment disparities in minority groups with multiple myeloma at a safety-net hospital [letter]. Leuk. Lymphoma https://doi.org/ 10.1080/10428194.2020.1767290 (2020)

16. Derman, B. A. et al. Racial differences in treatment and outcomes in multiple myeloma: a multiple myeloma research foundation analysis. Blood Cancer J. 10, 80 (2020).

17. Bhatnagar, V. et al. Disparities in Black and White patients with multiple myeloma referred for autologous hematopoietic transplantation: a single center study. Cancer 121, 1064-1070 (2015).

18. Costa, L. J. et al. Recent trends in multiple myeloma incidence and survival by age, race, and ethnicity in the United States. Blood Adv. 1, 282-287 (2017).

19. Ailawadhi, S. et al. Disease and outcome disparities in multiple myeloma: exploring the role of race/ethnicity in the Cooperative Group clinical trials. Blood Cancer J. 8, 67 (2018).

20. Fillmore, N. R. et al. With equal access, African American patients have superior survival compared to White patients with multiple myeloma: a VA study [letter]. Blood 133, 2615-2618 (2019).

21. Connors, L. H. et al. Cardiac amyloidosis in African Americans: comparison of clinical and laboratory features of transthyretin V122I amyloidosis and immunoglobulin light chain amyloidosis. Am. Heart J. 158, 607-614 (2009).

22. Jacobson, D. R., Alexander, A. A., Tagoe, C. \& Buxbaum, J. N. Prevalence of the amyloidogenic transthyretin (TTR) V122l allele in 14333 African-Americans. Amyloid 22, 171-174 (2015).

23. Connors, L. H. et al. A simple screening test for variant transthyretins associated with familial transthyretin amyloidosis using isoelectric focusing. Biochem. Biophys. Acta 1407, 185-192 (1998). 
24. Gertz, M. A. et al. Definition of organ involvement and treatment response in immunoglobulin light chain amyloidosis (AL): a consensus opinion from the 10th International Symposium on Amyloid and Amyloidosis, Tours, France, 1822 April 2004. Am. J. Hematol. 79, 319-328 (2005).

25. Lilleness, B., Ruberg, F. L., Mussinelli, R., Doros, G. \& Sanchorawala, V. Development and validation of a survival staging system incorporating BNP in patients with light chain amyloidosis. Blood 133, 215-223 (2019).

26. Palladini, G. et al. A staging system for renal outcome and early markers of renal response to chemotherapy in AL amyloidosis. Blood 124, 2325-2332 (2014).

27. Palladini, G. et al. New criteria for response to treatment in immunoglobulin light chain amyloidosis based on free light chain measurement and cardiac biomarkers: impact on survival outcomes. J. Clin. Oncol. 30, 4541-4549 (2012).

28. US Census Bureau. Annual Estimates of the Resident Population by Sex, Race, and Hispanic Origin for the United States: April 1, 2000 to July 1, 2009 (NCEST2009-03) (US Census Bureau, Washington, 2010).

29. Williams, D. R., Mohammed, S. A., Leavell, J. \& Collins, C. Race, socioeconomic status, and health: complexities, ongoing challenges, and research opportunities. Ann. NY Acad. Sci. 1186, 69-101 (2010).

30. Landgren, $\mathrm{O}$. et al. Obesity is associated with an increased risk of monoclonal gammopathy of undetermined significance among black and white women. Blood 116, 1056-1059 (2010).

31. Chang, S. H. et al. Obesity and the transformation of monoclonal gammopathy of undetermined significance to multiple myeloma: a population-based cohort study. J. Natl Cancer Inst. 109, djw264 (2016).

32. Birmann, B. M. et al. Young adult and usual adult body mass index and multiple myeloma risk: a pooled analysis in the International Multiple Myeloma Consortium (IMMC). Cancer Epidemiol. Biomarkers Prev. 26, 876-885 (2017).
33. Marinac, C. R. et al. Body mass index throughout adulthood, physical activity, and risk of multiple myeloma: a prospective analysis in three large cohorts. $\mathrm{Br}$. J. Cancer 118, 1013-1019 (2018).

34. Sonderman, J. S. et al. Multiple myeloma mortality in relation to obesity among African Americans. J. Natl Cancer Inst. 108, djw120 (2016).

35. Comenzo, R. L., Zhang, Y., Martinez, C., Osman, K. \& Herrera, G. A. The tropism of organ involvement in primary systemic amyloidosis: contributions of $\lg V(L)$ germ line gene use and clonal plasma cell burden. Blood 98, 714-720 (2001).

36. Perfetti, $\vee$. et al. The repertoire of lambda light chains causing predominant amyloid heart involvement and identification of a preferentially involved germline gene, IGLV1-44. Blood 119, 144-150 (2012).

37. Kourelis, T. V. et al. Clarifying immunoglobulin gene usage in systemic and localized immunoglobulin light-chain amyloidosis by mass spectrometry. Blood 129, 299-306 (2017).

38. Phull, $\mathrm{P}$. et al. Monoclonal gammopathy of undetermined significance in systemic transthyretin amyloidosis (ATTR). Amyloid 25, 62-67 (2018).

39. Donnelly, J. P. et al. Evidence of concurrent light chain and transthyretin cardiac amyloidosis in 2 patients. JACC CardioOncol. 2, 127-130 (2020).

40. Sanchorawala, $\mathrm{V}$. et al. Long-term outcome of patients with $\mathrm{AL}$ amyloidosis treated with high-dose melphalan and stem cell transplantation: 20-year experience. Blood 126, 2345-2347 (2015).

41. Sanchorawala, V. High-dose melphalan and autologous peripheral blood stem cell transplantation in AL amyloidosis. Acta Haematol. 143, 381-387 (2020).

42. Verma, P. S., Howard, R. S. \& Weiss, B. M. The impact of race on outcomes of autologous transplantation in patients with multiple myeloma. Am. J. Hematol. 83, 355-358 (2008)

43. Hamel, L. M. et al. Barriers to clinical trial enrollment in racial and ethnic minority patients with cancer. Cancer Control 23, 327-337 (2016). 